Received: 07.06.2019

Revised: 30.01 .2020

Accepted: 21.02 .2020

DOI: $10.17804 / 2410-9908.2020 .1 .034-042$

\title{
A METHOD FOR CALCULATING STRESSES IN A MULTIPLY CONNECTED ELASTIC BODY
}

\author{
V. V. Struzhanov \\ Institute of Engineering Science, Ural Branch of the Russian Academy of Sciences, \\ 34 Komsomolskaya St., Ekaterinburg, 620049, Russian Federation \\ (iD https://orcid.org/0000-0002-3669-2032《 stru@imach.uran.ru \\ Corresponding author. E-mail: stru@imach.uran.ru \\ Address for correspondence: 34 Komsomolskaya St., Ekaterinburg, 620049, Russian Federation \\ Tel.: +7(343) 3623019
}

An analytical method for determining the stress state in elastic bodies with a cavity is developed. The technique is based on using solutions to problems of the theory of elasticity for two simply connected regions, namely, for a body without a cavity and a space that is the exterior of a cavity. Special operator equations are obtained to determining the required stresses in a multiply connected body. An iterative method for solving these operator equations is proposed. A convergence of successive approximations is proved. An illustrative example is provided.

Keywords: multiply connected body, stress state, operator equation, successive approximation, iteration convergence.

\section{References}

1. Savin, G.N. and Tul'chii, V.I. Spravochnik po kontsentratsii napryazheniy [Handbook on Stress Concentrations]. Kiev, Vishcha Shkola Publ., 1976. (In Russian).

2. Savin G.N. Raspredelenie napryazheniy okolo otverstiy [Stress Distribution Around Holes]. Kiev, Naukova Dumka Publ., 1968, 891 p (In Russian).

3. Rabotnov Yu.N. Mekhanika devormirovannogo tverdogo tela [Mechanics of Deformable Solids]. M, Nauka Publ., 1988, 712 p. (In Russian).

4. Mirenkov V.E., Shutov V.A., Poluektov V.A. On the deformation of loosened plates. Izvestiya Vuzov, Stroitelstvo, 2002, no. 12, pp. 17-21. (In Russian).

5. Sil'vestrov V.V., Zemlyanova A.Yu. Repair of a Plate with a Circular Hole by Applying a Patch. Journal of Applied Mechanics and Technical Physics, 2004, vol. 45, no. 4, pp. 605-611. DOI: 10.1023/B:JAMT.0000030342.06634.ec.

6. Levshchanova L.L. The destruction of the coating on a plate with a cutout. Mekhanika kompozitsionnykh materialov $i$ konstruktsii (Mechanics of Composite Materials and Structures), 2007, vol. 13, no. 2, pp. 233-238. (In Russian).

7. Mokryakov V.V. The use of the multipole format for solving problems of two close located holes. Mechanics of Solids, 2007, vol. 42, iss. 5, pp 771-785. DOI: 10.3103/S0025654407050111.

8. Kudryavtsev S.V. Kontsentratsiya naprryazheniy vblizi krugovykh otverstiy $v$ gofrirovannykh stenkakh balok [Stress Concentration Near Circular Holes in Corrugated Beam Walls]. Ekaterinburg, AMB Publishing House, 2010, 156 p. (In Russian).

9. Khan Kh. Teoriya uprugosti [Theory of Elasticity]. Moscow, Mir Publ., 1988, 344 p. (In Russian).

10. Lurie A.I. Teoriya uprugosti [Theory of Elasticity]. Moscow, Nauka Publ., 1970, 940 p. (In Russian). 
11. Dmitrienko Yu.I. Tenzornoe ischislenie [Tensor Calculation]. Moscow, Vysshaya Shkola Publ., 2001, 575 p. (In Russian).

12. Mikhlin S.G. Variatsionnye metody $v$ matematicheskoy fizike [Variational Methods in Mathematical Physics]. Moscow, Nauka Publ., 1970, 512 p. (In Russian).

13. Lyusternik L.A, Sobolev V.I. Elementy funktsionalnogo analiza [Elements of Functional Analysis]. Moscow, Nauka Publ., 1965, 520 p. (In Russian).

14. Timoshenko S., Gudier J.N. Teoriya uprugosti, Rus. transl. [Theory of Elasticity, New York, Toronto, London, McGraw-Hill Book Company, 1951]. Moscow, Nauka Publ., 1971. (In Russian). 
Подана в журнал: 07.06.2020

УДК 539.3

DOI: $10.17804 / 2410-9908.2020 .1 .034-42$

\title{
ОБ ОДНОМ МЕТОДЕ РАСЧЕТА НАПРЯЖЕНИЙ В НЕОДНОСВЯЗНОМ УПРУГОМ ТЕЛЕ
}

\author{
В. В. Стружанов
}

\author{
Федеральное государственное бюджетное учреждение науки \\ Институт машиноведения Уральского отделения Российской академии наук, \\ ул. Комсомольская, 34, Екатеринбург, Российская Федерация \\ (iD https://orcid.org/0000-0002-3669-2032 stru@imach.uran.ru
}

\author{
Ответственный автор. Электронная почта: stru@imach.uran.ru \\ Адрес для переписки: ул. Комсомольская, 34, Екатеринбург, Российская Федерация \\ Тел.: +7(343) 362-30-19
}

Разработан аналитический метод определения напряженного состояния в упругих телах с полостью. Методика основана на использовании решений задач теории упругости для двух односвязных областей, а именно, для тела без полости и пространства, являющегося внешностью полости. Получены специальные операторные уравнения, решения которых определяют искомые напряжения в неодносвязном теле. Предложен итерационный метод решения данных операторных уравнений. Доказана сходимость последовательных приближений. Приведен иллюстрированный пример.

Ключевые слова: неодносвязное тело, напряженное состояние, операторное уравнение, последовательные приближения, сходимость итераций.

\section{1. Введение}

Многие элементы конструкций имеют отверстия конструктивного или технологического назначения. Для оценки надежности, прочности и долговечности таких конструктивных элементов необходимо знать их напряженное состояние, поскольку оно существенно неоднородное и возле отверстий появляются зоны концентрации напряжений, где и начинается процесс разрушения [1-3]. Из последних работ по этой тематике можно отметить исследования [4-8]. Таким образом, отверстия (полости) оказывают определяющее влияние на работоспособность изделий. Поэтому для оценки прочности деталей необходимо решать краевые задачи теории упругости для неодносвязных областей, на внешних границах которых заданы силы. С практической точки зрения возможно воспользоваться различными численными методами, например методом конечных элементов. Однако для понимания свойств уравнений и полученных решений, а также для тестирования численных методов необходимо во многих случаях иметь аналитические решения. Особенно, когда коэффициент концентрации напряжений слишком велик. В работе предложен алгоритм расчета напряжений в упругих телах с полостями. Построены соответствующие операторные уравнения, разработан итерационный метод их решения и доказана сходимость итераций к точному аналитическому решению задачи.

\section{2. Постановка задачи}

Пусть в трехмерном евклидовом пространстве $E^{3}$ имеются два односвязных тела: $V_{1}$, ограниченное кусочно-гладкой поверхностью $\Gamma_{1}$, и $V_{2}$, ограниченное кусочно-гладкой поверхностью $\Gamma_{2}$. Причем $V_{2} \subset V_{1}$. Составим неодносвязное тело $V=V_{1} \backslash V_{2}$, т. е. $V_{2}-$ это полость в области $V_{1}$ (рисунок). 


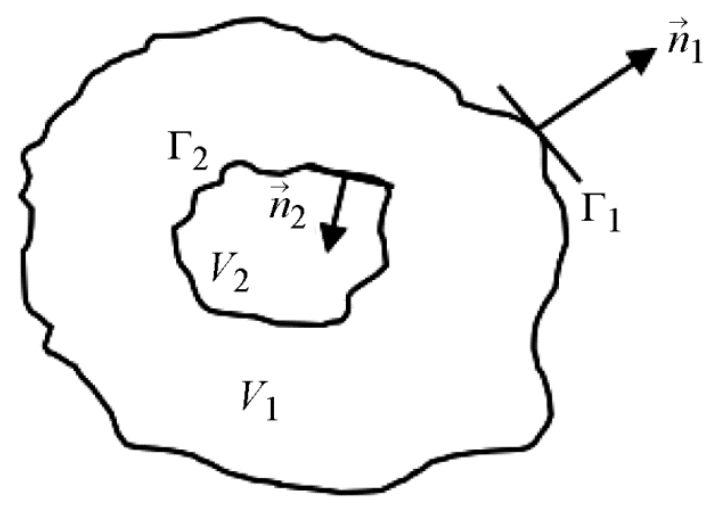

Неодносвязное тело

Свойства материала в данных областях определяются однородным изотропным симметричным тензором четвертого ранга модулей упругости $C$ [9]. В дальнейшем полагаем, что этот тензор распространен на все пространство $E^{3}$. Требуется найти напряженное состояние в неодносвязном теле $V$, если на его границах $\Gamma_{1}$ и $\Gamma_{2}$ заданы системы уравновешенных внешних сил, соответственно $t_{1}$ и $t_{2}$, объемные силы отсутствуют.

Напряженно-деформированное состояние в теле $V$ определяется решением системы уравнений краевой задачи теории упругости, которая в инвариантной форме имеет вид [10]:

$$
\nabla \cdot \sigma=0 ; \varepsilon=\operatorname{def} u ; \quad \sigma=C \cdot \varepsilon,
$$

с граничными условиями

$$
\left.\sigma \cdot n_{1}\right|_{\Gamma_{1}}=t_{1} ;\left.\quad \sigma \cdot n_{2}\right|_{\Gamma_{2}}=t_{2}
$$

Здесь первая группа уравнений - это уравнения равновесия; $\sigma$ - симметричный тензор второго ранга напряжений; $\nabla$ - оператор Гамильтона [6], точкой обозначено скалярное произведение тензора напряжений на оператор $\nabla$ (вектор Гамильтона). Вторая группа уравнений - соотношения Коши ( $\varepsilon$ - симметричный тензор второго ранга деформаций; $u$ - вектор перемещений). Третья группа уравнений - это закон Гука, связывающий тензоры напряжений и деформаций (двумя точками обозначено двойное скалярное произведение тензора четвертого ранга с тензором второго ранга $[10,11])$. Вектор $n_{1}$ - единичный вектор внешней нормали к поверхности $\Gamma_{1} ; n_{2}$ - единичный вектор внешней нормали к поверхности $\Gamma_{2}$, направленный в сторону области $V_{2}$. Точкой обозначено скалярное произведение тензора напряжений на вектор внешней нормали.

\section{3. Метод решения}

Пусть известны алгоритмы решений системы уравнений (1) для односвязной области $V_{1}$ с произвольными граничными условиями на поверхности $\Gamma_{1}$, а именно $\left.\sigma \cdot n_{1}\right|_{\Gamma_{1}}=g_{n}$ (или $\sigma \cdot n_{1}=g_{n}$ ), и для области $V_{3}=E^{3} \backslash V_{2}$ (внешность поверхности $\Gamma_{2}$ ) с произвольными условиями на поверхности $\Gamma_{2}$, а именно $\left.\sigma \cdot n_{2}\right|_{\Gamma_{2}}=p_{m}$ (или $\sigma \cdot n_{2}=p_{m}$ ). То есть, известны линейные отображения (операторы)

$$
A_{1}: g_{n} \rightarrow \sigma_{1}^{n} ; \quad A_{2}: p_{m} \rightarrow \sigma_{2}^{m},
$$

где $\sigma_{1}^{n}$ и $\sigma_{2}^{m}-$ тензоры напряжений, являющиеся решениями соответствующих краевых задач для областей $V_{1}$ и $V_{3}$. Решение сформулированной выше задачи для несвязанной области $V$ можно представить в виде суммы: 


$$
\sigma(x)=\sigma_{1}^{\prime}(x)+\sigma_{2}^{\prime}(x), \quad x \in V,
$$

где тензоры напряжений должны быть таковы, что выполняются условия

$$
\begin{aligned}
& n_{1} \cdot \sigma_{1}^{\prime}+n_{1} \cdot \sigma_{2}^{\prime}=t_{1} \\
& n_{2} \cdot \sigma_{1}^{\prime}+n_{2} \cdot \sigma_{2}^{\prime}=t_{2} .
\end{aligned}
$$

Здесь тензоры $\sigma_{1}^{\prime}$ и $\sigma_{2}^{\prime}-$ некоторые (пока неизвестные) решения краевой задачи (1) соответственно для областей $V_{1}$ и $V_{3}$.

Получим теперь уравнения для определения $\sigma_{1}^{\prime}$ и $\sigma_{2}^{\prime}$. Применим операторы $A_{1}$ и $A_{2}$ соответственно к условиям (2) и (3):

$$
\begin{aligned}
& A_{1}\left(n_{1} \cdot \sigma_{1}^{\prime}\right)+A_{1}\left(n_{1} \cdot \sigma_{2}^{\prime}\right)=A_{1} t_{1} \\
& A_{2}\left(n_{2} \cdot \sigma_{1}^{\prime}\right)+A_{2}\left(n_{2} \cdot \sigma_{2}^{\prime}\right)=A_{2} t_{2} .
\end{aligned}
$$

Тогда учитывая, что $A_{1}\left(n_{1} \cdot \sigma_{1}^{\prime}\right)=\sigma_{1}^{\prime} ; A_{2}\left(n_{2} \cdot \sigma_{2}^{\prime}\right)=\sigma_{2}^{\prime}$, получаем:

$$
\begin{aligned}
& \sigma_{1}^{\prime}+B_{1} \sigma_{2}^{\prime}=S_{1}, \quad x \in V_{1} ; \\
& \sigma_{2}^{\prime}+B_{2} \sigma_{1}^{\prime}=S_{2}, \quad x \in V_{3} .
\end{aligned}
$$

где $B_{1} \sigma_{2}^{\prime}=A_{1}\left(n_{1} \cdot \sigma_{2}^{\prime}\right) ; \sigma_{1}^{\prime}=A_{2}\left(n_{2} \cdot \sigma_{1}^{\prime}\right) ; A_{1} t_{1}=S_{1}-$ тензор напряжений, являющийся решением краевой задачи (1) для области $V_{3}$ с граничными условиями $t_{2}$.

Подставляя выражение $\sigma_{1}^{\prime}$ из уравнения (4) в уравнение (5), а выражение $\sigma_{2}^{\prime}$ из уравнения (5) - в уравнение (4), находим два независимых уравнения для определения $\sigma_{1}^{\prime}$ и $\sigma_{2}^{\prime}$, а именно:

$$
\begin{aligned}
& \sigma_{1}^{\prime}=B_{1} B_{2} \sigma_{1}^{\prime}+\left(S_{1}-B_{1} S_{2}\right), \quad x \in V_{1} ; \\
& \sigma_{2}^{\prime}=B_{2} B_{1} \sigma_{2}^{\prime}+\left(S_{2}-B_{2} S_{1}\right), \quad x \in V_{3} .
\end{aligned}
$$

Решения уравнений (6) и (7) будем искать методом последовательных приближений:

$$
\begin{aligned}
& \sigma_{1}^{\prime}=\sum_{k=0}^{\infty}\left(B_{1} B_{2}\right)^{k}\left(S_{1}-B_{1} S_{2}\right) ; \\
& \sigma_{2}^{\prime}=\sum_{k=0}^{\infty}\left(B_{2} B_{1}\right)^{k}\left(S_{2}-B_{2} S_{1}\right) .
\end{aligned}
$$

Покажем, что операторы $B_{1} B_{2}$ и $B_{2} B_{1}$ есть операторы сжатия в соответствующих функциональных пространствах. Возьмем сначала энергетическое вещественное гильбертово пространство $T_{1}\left(V_{1}\right)$ тензоров напряжений, определенных в односвязной области $V_{1}$ и связанные законом Гука с тензорами деформаций, удовлетворяющих условиям совместности 
[12]. Если тензор $q \in T_{1}\left(V_{1}\right)$, то $q \cdot S \cdot q$ - положительно определенная форма, значение которой равно удвоенной потенциальной энергии упругих деформаций элемента материала. Норма в энергетическом пространстве $T_{1}\left(V_{1}\right)$ определяется выражением $\|q\|_{T_{1}\left(V_{1}\right)}^{2}=$ $\int_{V_{1}} q \cdot s \cdot q d V$ [12] (это удвоенная потенциальная энергия упругих деформаций всего тела $\left.V_{1}\right)$. Здесь $S=C^{-1}$ - тензор четвертого ранга модулей податливости. Отметим, что тензоры напряжений в уравнении (6) есть тензоры из $T_{1}\left(V_{1}\right)$.

Оценим норму оператора $B_{1} B_{2}$ в пространстве $T_{1}\left(V_{1}\right)$. Используем теорему Клайперона при отсутствии объемных сил, записанную в инвариантной форме [10,12]:

$$
\int_{W} q \cdot S \cdot q d V=\int_{\Gamma} u \cdot(n \cdot q) d \Gamma .
$$

Здесь $W$ односвязное тело с границей $\Gamma ; ~ u-$ вектор перемещений; $n$ - единичный вектор внешней нормали. Тогда

$$
\begin{gathered}
\|q\|_{T_{1}\left(V_{1}\right)}^{2}=\int_{V_{1}} q \cdot S \cdot q d V>\int_{V_{2}} q \cdot S \cdot q d V= \\
=\int_{\Gamma_{2}} u_{2} \cdot\left(-n_{2} \cdot q\right) d \Gamma=\int_{V_{3}} A_{2}\left(-n_{2} \cdot q\right) \cdot S \cdot A_{2}\left(-n_{2} \cdot q\right) d V> \\
>\int_{E^{3} \backslash V_{1}} A_{2}\left(-n_{2} \cdot q\right) \cdot S \cdot A_{2}\left(-n_{2} \cdot q\right) d V= \\
=\int_{\Gamma_{1}} u_{1}\left[-n_{1} \cdot A_{2}\left(-n_{2} \cdot q\right)\right] d \Gamma=\int_{V_{1}} A_{1}\left[n_{1} \cdot A_{2}\left(n_{2} \cdot q\right)\right] \cdot S \cdot A_{1}\left[n_{1} \cdot A_{2}\left(n_{2} \cdot q\right)\right] d V= \\
=\left\|B_{1} B_{2} q\right\|_{T_{1}\left(V_{1}\right)}^{2} \cdot
\end{gathered}
$$

Отсюда $\|q\|_{T_{1}\left(V_{1}\right)}^{2}>\left\|B_{1} B_{2} q\right\|_{T_{1}\left(V_{1}\right)}^{2}$. Здесь $u_{1}, u_{2}$ - векторы перемещений, заданные соответственно на границах $\Gamma_{1}$ и $\Gamma_{2}$. Отметим, что интегралы по областям $V_{3}$ и $E^{3} \backslash V_{1}$ имеют конечные значения, так как тензоры напряжений (решения краевой задачи (1) для внешней поверхности $\Gamma_{1}$ и $\Gamma_{2}$ ) достаточно быстро убывают и обращаются в нуль на бесконечности. Теперь [13]

$$
\left\|B_{1} B_{2}\right\|_{T_{1}\left(V_{1}\right)}=\sup \frac{\left\|B_{1} B_{2} q\right\|_{T_{1}\left(V_{1}\right)}}{\|q\|_{T_{1}\left(V_{1}\right)}}<1
$$

Следовательно, оператор $B_{1} B_{2}$ есть оператор сжатия для элементов пространства $T_{1}\left(V_{1}\right)$ и ряд (8) сходится по норме пространства $T_{1}\left(V_{1}\right)$ к решению уравнения (6).

Возьмем теперь энергетическое вещественное гильбертово пространство $T_{1}\left(V_{3}\right)$ тензоров напряжений, определенных в области $E^{3} \backslash V_{1}$ и связанных законом Гука с тензорами деформаций, удовлетворяющих условиям совместности. Если $q \in T_{1}\left(V_{1}\right)$, то $q \cdot S \cdot q-$ положительно определенная форма. Норма в пространстве $T_{1}\left(V_{3}\right)$ есть $\|q\|_{T_{1}\left(V_{3}\right)}^{2}=$ $\int_{V_{3}} q \cdot S \cdot q d V$ (это удвоенная потенциальная энергия тела $V_{3}$ ). Аналогично изложенному выше оценим норму оператора $B_{2} B_{1}$. Имеем: 


$$
\begin{aligned}
& =\int_{V_{3}} q \cdot S \cdot \cdot q d V>\int_{E^{3} \backslash V_{1}} q \cdot S \cdot \cdot q d V= \\
& =\int_{\Gamma_{1}} u_{1} \cdot\left(-n_{1} \cdot q\right) d \Gamma=\int_{V_{1}} A_{1}\left(-n_{1} \cdot q\right) \cdot S \cdot A_{1}\left(-n_{1} \cdot q\right) d V> \\
& >\int_{V_{2}} A_{1}\left(-n_{1} \cdot q\right) \cdot S \cdot A_{1}\left(-n_{2} \cdot q\right) d V= \\
& =\int_{\Gamma_{2}} u_{2}\left[-n_{2} \cdot A_{1}\left(-n_{1} \cdot q\right)\right] d \Gamma=\int_{V_{3}} A_{2}\left[n_{2} \cdot A_{1}\left(n_{1} \cdot q\right)\right] \cdot S \cdot A_{2}\left[n_{2} \cdot A_{1}\left(n_{1} \cdot q\right)\right] d V= \\
& =\left\|B_{2} B_{1} q\right\|_{T_{1}\left(V_{3}\right)}^{2} . \\
& \text { Отсюда }\|q\|_{T_{1}\left(V_{3}\right)}^{2}>\left\|B_{2} B_{1} q\right\|_{T_{1}\left(V_{3}\right)}^{2} \text {. } \\
& \text { Тогда }\left\|B_{2} B_{1}\right\|_{T_{1}\left(V_{3}\right)}=\sup _{\|q\| \neq 0} \frac{\left\|B_{1} B_{2} q\right\|_{T_{1}\left(V_{1}\right)}}{\|q\|_{T_{1}\left(V_{1}\right)}}<1 .
\end{aligned}
$$

Таким образом, оператор $B_{2} B_{1}$ есть оператор сжатия и ряд (9) сходится по норме пространства $T_{1}\left(V_{3}\right)$ к решению уравнения (7). Наконец, складывая решения уравнений (6) и (7), находим решение исходной задачи, т. е. напряжения в неодносвязном упругом теле $V$ при заданных внешних силах (векторах $t_{1}$ и $t_{2}$ ).

\section{4. Тестовый пример}

Применим изложенную методику для решения тестовой задачи о расчете напряжений в толстостенной трубе с внешним радиусом $b$ и внутренним радиусом $a$, находящейся под воздействием внешнего и внутреннего давлений соответственно с интенсивностями $p_{1}$ и $p_{2}$ (задача Ляме). Граничные условия в этом случае равны $t_{1}=\left.\sigma_{r}\right|_{r=b}=-p_{1}, t_{2}=\left.\sigma_{r}\right|_{r=a}=-p_{2}$. В данной задаче область $V_{1}$ - это сплошной круговой цилиндр с радиусом основания $b$, на который действует равномерное внешнее давление интенсивностью $t_{1}$. Область $V_{3}-$ это трехмерное пространство с цилиндрическим отверстием с радиусом $a$, внутри которого создано давление с интенсивностью $t_{2}$. Используя известные решения для областей $V_{1}$ и $V_{3}$ с определенными выше граничными условиями $[9,10]$, получаем:

$$
\begin{gathered}
S_{1}=A_{1}\left(-p_{1}\right)=\left|\begin{array}{ccc}
-p_{1} & 0 & 0 \\
0 & -p_{1} & 0 \\
0 & 0 & -2 v p_{1}
\end{array}\right|, \quad S_{2}=A_{2}\left(-p_{2}\right)=\left|\begin{array}{ccc}
-p_{2} & 0 & 0 \\
0 & -p_{2} & 0 \\
0 & 0 & 0
\end{array}\right| \frac{a^{2}}{r^{2}} ; \\
B_{1} S_{2}=\left|\begin{array}{ccc}
-p_{2} & 0 & 0 \\
0 & -p_{2} & 0 \\
0 & 0 & -2 v p_{2}
\end{array}\right| \frac{a^{2}}{b^{2}}, \quad B_{2} S_{1}=\left|\begin{array}{ccc}
-p_{1} & 0 & 0 \\
0 & p_{1} & 0 \\
0 & 0 & 0
\end{array}\right| \frac{a^{2}}{r^{2}} ; \\
S_{1}-B_{1} S_{2}=\left|\begin{array}{ccc}
-p_{1} & 0 & 0 \\
0 & -p_{1} & 0 \\
0 & 0 & -2 v p_{1}
\end{array}\right|-\left|\begin{array}{ccc}
-p_{2} & 0 & 0 \\
0 & -p_{2} & 0 \\
0 & 0 & -2 v p_{2}
\end{array}\right| \frac{a^{2}}{b^{2}}
\end{gathered}
$$


Здесь $v$ - коэффициент Пуассона, на главной диагонали тензоров соответствующих напряжений сверху вниз расположены радиальные, тангенциальные и осевые напряжения. Отсюда ряды (8) и (9) соответственно имеют вид:

$$
\begin{aligned}
& \sigma_{1}^{\prime}=L+L \frac{a^{2}}{b^{2}}+L \frac{a^{4}}{b^{4}}+\cdots=\frac{L b^{2}}{b^{2}-a^{2}} \\
& \sigma_{2}^{\prime}=K+K \frac{a^{2}}{b^{2}}+K \frac{a^{4}}{b^{4}}+\cdots=\frac{K b^{2}}{b^{2}-a^{2}}
\end{aligned}
$$

где

$$
\begin{gathered}
L=\left|\begin{array}{ccc}
-p_{1} & 0 & 0 \\
0 & -p_{1} & 0 \\
0 & 0 & -2 v p_{1}
\end{array}\right|+\left|\begin{array}{ccc}
p_{2} & 0 & 0 \\
0 & p_{2} & 0 \\
0 & 0 & 2 v p_{2}
\end{array}\right| \frac{a^{2}}{b^{2}} ; \\
K=\left|\begin{array}{ccc}
-p_{2} & 0 & 0 \\
0 & p_{2} & 0 \\
0 & 0 & 0
\end{array}\right| \frac{a^{2}}{r^{2}}-\left|\begin{array}{ccc}
p_{1} & 0 & 0 \\
0 & -p_{1} & 0 \\
0 & 0 & 0
\end{array}\right| \frac{a^{2}}{r^{2}} .
\end{gathered}
$$

Теперь искомый тензор напряжений $\sigma=\sigma_{1}^{\prime}+\sigma_{2}^{\prime}(a \leq r \leq b)$. Нетрудно убедиться, что после подстановки значений (10) и (11) получаем известное решение Ляме [14].

\section{5. Заключение}

Построены операторные уравнения, решения которых определяют напряженное состояние в упругих областях с полостями. Разработан метод последовательных приближений их решения. Доказана сходимость итераций к точному решению задачи теории упругости для неодносвязного тела.

\section{Литература}

1. Савин Г. Н., Тульчий В. И. Справочник по концентрации напряжений. - Киев : Вища школа, 1976. $-412 \mathrm{c.}$

2. Савин Г. Н. Распределение напряжений около отверстий. - Киев : Наукова думка, 1968. - 891c.

3. Работнов Ю. Н. Механика деформируемого твердого тела. - М. : Наука, 1988. - 712 с.

4. Миренков В. Е., Шутов В. А., Полуэктов В. А. О деформировании пластин с ослаблениями // Известия вузов. Строительство. - 2002. - № 12. - С. 17-21.

5. Sil'vestrov V. V., Zemlyanova A.Yu. Repair of a plate with a circular hole by applying a patch // Journal of Applied Mechanics and Technical Physics. - 2004. - Vol. 45, no. 4. P. 605-611. - DOI: 10.1023/B:JAMT.0000030342.06634.ec.

6. Левщанова Л. Л. Разрушение покрытий на пластинах с вырезом // Механика композитных материалов и конструкций. - 2007. - Т. 13, № 2. - С. 233-238.

7. Mokryakov V. V. The use of the multipole format for solving problems of two close located holes // Mechanics of Solids. - 2007. - Vol. 42, iss. 5. - P 771-785. DOI: $10.3103 / \mathrm{S} 0025654407050111$.

8. Кудрявцев С. В. Концентрация напряжений вблизи круговых отверстий в гофрированных стенках балок. - Екатеринбург : Изд-во АМБ. - 2010. - 156 с.

9. $\quad$ Хан Х. Теория упругости. - М : Мир, 1988. - 344 с.

10. Лурье А. И. Теория упругости. - М : Наука, 1970. - 940 с.

11. Дмитриенко Ю. И. Тензорное исчисление. - М : Высшая школа, 2001. - 575 с. 
12. Михлин С. Г. Вариационные методы в математической физике. - М : Наука, 1970. $512 \mathrm{c}$.

13. Люстерник Л. А., Соболев В. И. Элементы функционального анализа. - М : Наука, 1965. $-520 \mathrm{c}$.

14. Тимошенко С. П., Гудьер Дж. Теория упругости / пер. с англ. - М : Наука, 1971. $560 \mathrm{c}$. 